FTUV/95-31

IFIC/95-33

hep-th/9512187

\title{
Solvable Models for radiating Black Holes and Area-preserving Diffeomorphisms内
}

\author{
J.Cruz Tand J. Navarro-Salasf \\ Departamento de Física Teórica and \\ IFIC, Centro Mixto Universidad de Valencia-CSIC. \\ Facultad de Física, Universidad de Valencia, \\ Burjassot-46100, Valencia, Spain.
}

March 25, 2022

\begin{abstract}
Solvable theories of 2D dilaton gravity can be obtained from a Liouville theory by suitable field redefinitions. In this paper we propose a new framework to generate $2 \mathrm{D}$ dilaton gravity models which can also be exactly solved in the semiclassical approximation. Our approach is based on the recently introduced scheme to quantize massless scalar fields coupled to 2D gravity maintaining invariance under area-preserving diffeomorphisms and Weyl transformations. Starting from the CGHS model with the new effective action we reestablish the full diffeomorphism invariance by means of an adequate family of field redefinitions. The original theory is therefore mapped into a large family of solvable models.We focus our analysis on the one-parameter class of models interpolating between the Russo-Susskind-Thorlacius model and the Bose-Parker-Peleg model. Finally we shall briefly indicate how can we extend our approach to spherically symmetric Einstein gravity coupled to 2D conformal matter.
\end{abstract}

\footnotetext{
${ }^{*}$ Work partially supported by the Comisión Interministerial de Ciencia y Tecnología and DGICYT.

${ }^{\dagger}$ CRUZ@LIE.IFIC.UV.ES

‡JNAVARRO@LIE.IFIC.UV.ES
} 


\section{Introduction}

One of the most interesting features of $2 \mathrm{D}$ dilaton black holes is the possibility of constructing solvable models for the evaporation process including the backreaction effects. The solvability of the models can be traced back to the fact that, in conformal gauge, a set of field redefinitions of the metric-dilaton fields convert the gravity models into a Liouville theory [四. A simple model of this type was provided by Russo, Susskind and Thorlacius [2]. The addition of the counterterm

$$
-\frac{N}{96 \pi} \int d^{2} x \sqrt{-g} 2 \phi R
$$

to the standard one-loop effective action of the Callan-Giddings-Harvey- Strominger (CGHS) model [3] $S_{0}+S_{P}$, where

$$
S_{0}=\frac{1}{2 \pi} \int d^{2} x \sqrt{-g}\left[e^{-2 \phi}\left(R+4(\nabla \phi)^{2}+4 \lambda^{2}\right)-\frac{1}{2} \sum_{i=1}^{N}\left(\nabla f_{i}\right)^{2}\right],
$$

is the classical CGHS action and $S_{P}$ is the Polyakov action

$$
S_{P}=-\frac{N}{96 \pi} \int d^{2} x \sqrt{-g} R \square^{-1} R,
$$

restores the solvability of the classical theory. The field redefinitions $\left(k=\frac{N}{12}\right)$ :

$$
\begin{gathered}
\Omega=\frac{\sqrt{k}}{2} \phi+\frac{e^{-2 \phi}}{\sqrt{k}}, \\
\chi=\sqrt{k} \rho-\frac{\sqrt{k}}{2} \phi+\frac{e^{-2 \phi}}{\sqrt{k}},
\end{gathered}
$$

convert the RST model [2] into a Liouville theory

$$
\frac{1}{\pi} \int d^{2} x\left[-\partial_{+} \chi \partial_{-} \chi+\partial_{+} \Omega_{-} \partial_{-} \Omega^{2} e^{\frac{2}{\sqrt{k}}(\chi-\Omega)}+\frac{1}{2} \sum_{i=1}^{N} \partial_{+} f_{i} \partial_{-} f_{i}\right]
$$

Under general assumptions: i) the absence of unphysical fluxes at infinity in the vacuum, ii) the existence of a vacuum solution with $R=0$, the most general semiclassical theory, containing the standard Polyakov term, that can be cast into the form (1.6) is given by the one-parameter class of models [4]

$$
\begin{aligned}
& S=\frac{1}{2 \pi} \int d^{2} x \sqrt{-g}\left[e^{-\frac{2}{n} \phi}\left(R+\frac{4}{n}(\nabla \phi)^{2}\right)+4 \lambda^{2} e^{-2 \phi}-\frac{1}{2} \sum_{i=1}^{N}\left(\nabla f_{i}\right)^{2}\right. \\
& \left.+k\left(\frac{1-2 n}{2 n} \phi R+\frac{(n-1)}{n}(\nabla \phi)^{2}-\frac{1}{4} R \square^{-1} R\right)\right]
\end{aligned}
$$

where the different models are labelled by the parameter $n$. 
The above family of dilaton-gravity models is however rather restrictive. The interesting case of Einstein theory -restricted to spherical field configurationscoupled to null matter $\left({ }^{(4)} d s^{2}=g_{\mu \nu} d x^{\mu} d x^{\nu}+\frac{e^{-2 \phi}}{\lambda^{2}} d \Omega^{2} \mu, \nu=1,2\right)$

$$
\frac{1}{2 \pi} \int d^{2} x \sqrt{-g}\left[e^{-2 \phi}\left(R+2(\nabla \phi)^{2}+2 \lambda^{2} e^{2 \phi}\right)-\frac{1}{2} \sum_{i=1}^{N}\left(\nabla f_{i}\right)^{2}\right],
$$

cannot be reached by this framework.

In this paper we want to put forward an alternative approach to construct exactly solvable models by extending the results of Ref [5]. In section 2 we introduce the basic ingredients of the Weyl-invariant quantization scheme for two-dimensional massless fields. In section 3 we describe a procedure of field redefinitions to generate exactly solvable semiclassical models for the CGHS theory. Section 4 is devoted to study the one-parameter class of solvable models which continuously interpolates between the RST model and the Bose-ParkerPeleg (BPP) model [6]. In section 5 we generalize the structure of the field redefinitions of the CGHS theory to a general class of dilaton-gravity models and define the analogue of the RST and BPP models for spherically symmetric gravity.

\section{Area-preserving diffeomorphisms and the Virasoro anomaly}

The Polyakov effective action $S_{P}$ in (1.2) reflects the well known fact that the expectation value of the stress tensor operator of $\mathrm{N}$ massless scalar fields coupled to gravity in two dimensions verify the equations:

$$
\begin{gathered}
\nabla_{\mu}<T^{f \mu \nu}>=0, \\
<T_{\mu}^{f \mu}>=\frac{N}{24} R .
\end{gathered}
$$

The covariant conservation equation (2.1) is a consequence of the covariance in the choice of the functional measure for the conformal fields:

$$
\left\|\delta f_{i}\right\|^{2}=\int d^{2} x \sqrt{-g} \delta f_{i} \delta f_{i} .
$$

The anomaly relation (2.2) reflects the breaking of Weyl symmetry in the quantization procedure. One can alternatively propose a different way of defining the functional measure that preserve Weyl invariance [7, 8] (see also [9] ).

$$
\left\|\delta f_{i}\right\|^{2}=\int d^{2} x \delta f_{i} \delta f_{i}
$$

Diffeomorphism invariance is therefore lost although invariance under areapreserving diffeomorphisms is maintained (see [10] for a general study). This leads to a new effective action $S_{W}$ for conformal matter

$$
S_{W}\left(g^{\mu \nu}\right)=S_{P}\left(\sqrt{-g} g^{\mu \nu}\right)
$$


and the new effective stress tensor

$$
T_{\mu \nu}^{W}=-\frac{2 \pi}{\sqrt{-g}} \frac{\delta S_{W}}{\delta g^{\mu \nu}},
$$

satisfies the anomaly relations

$$
\begin{gathered}
T_{\mu}^{W \mu}=0 \\
\nabla^{\mu} T_{\mu \nu}^{W}=-\frac{N}{48} \frac{1}{\sqrt{-g}} \partial_{\nu} R\left(\sqrt{-g} g^{\mu \nu}\right),
\end{gathered}
$$

In conformal gauge, $d s^{2}=-e^{2 \rho} d x^{+} d x^{-}$, the above expressions take a simple form

$$
\begin{gathered}
T_{+-}^{W}=0, \\
\nabla^{+} T_{++}^{W}=\nabla^{-} T_{--}^{W}=0 .
\end{gathered}
$$

$T_{\mu \nu}^{W}$ transforms covariantly under area-preserving diffeomorphisms, but under general conformal coordinate transformations $x^{ \pm} \longrightarrow y^{ \pm}\left(x^{ \pm}\right) T_{ \pm \pm}^{W}$ transforms according to the Virasoro anomaly:

$$
T_{x^{ \pm} x^{ \pm}}^{W}=\left(\frac{d y^{ \pm}}{d x^{ \pm}}\right)^{2} T_{y^{ \pm} y^{ \pm}}^{W}-\frac{N}{24}\left\{y^{ \pm}, x^{ \pm}\right\},
$$

where $\{y, x\}$ is the schwartzian derivative.

\section{Field redefinitions and solvable models for the CGHS theory}

The relationship between the non-covariant scheme defined by the "semiclassical" action

$$
S_{0}+S_{W}
$$

and the standard (covariant) approach was investigated in Ref [5]. By relating in conformal gauge the solutions of the non-covariant theory with the classical solutions in vacuum it was found a set of field redefinitions which convert the theory (3.1) into the RST model . The field redefinitions are:

$$
\begin{gathered}
\rho-\phi=\hat{\rho}-\hat{\phi} \\
e^{-2 \phi}=e^{-2 \hat{\phi}}+\frac{N}{24} \hat{\phi}+\frac{N}{12} M(\hat{\rho}-\hat{\phi}),
\end{gathered}
$$

where

$$
\begin{aligned}
M(\hat{\rho}-\hat{\phi})= & \hat{\rho}-\hat{\phi}+\int^{y^{+}} e^{2(\hat{\rho}-\hat{\phi})} \int e^{-2(\hat{\rho}-\hat{\phi})}\left[\partial_{y^{+}}(\hat{\rho}-\hat{\phi})\right]^{2} \\
& +\int^{y^{-}} e^{2(\hat{\rho}-\hat{\phi})} \int e^{-2(\hat{\rho}-\hat{\phi})}\left[\partial_{y^{-}}(\hat{\rho}-\hat{\phi})\right]^{2}
\end{aligned}
$$

$(\phi, \rho)$ and $(\hat{\phi}, \hat{\rho})$ refer to the non-covariant and covariant theories respectively. 
Now we shall further explore the structure of the above field redefinitions. The anomalous transformation properties of the fields $(\phi, \rho)$ can be read immediately from the form of the classical solution

$$
\begin{aligned}
e^{-2 \phi}= & -\int^{y^{+}} e^{2(\rho-\phi)} \int e^{-2(\rho-\phi)}\left(T_{++}^{c l}+T_{++}^{W}\right) \\
& -\int^{y^{-}} e^{2(\rho-\phi)} \int e^{-2(\rho-\phi)}\left(T_{--}^{c l}+T_{--}^{W}\right) \\
& -\lambda^{2} e^{-2(\rho-\phi)} \int^{y^{+}} e^{2(\rho-\phi)} \int^{y^{-}} e^{2(\rho-\phi)} .
\end{aligned}
$$

The Virasoro anomaly of $T_{ \pm \pm}^{W}$ makes that, under the conformal transformations $y^{ \pm} \longrightarrow z^{ \pm}\left(y^{ \pm}\right), e^{-2 \phi}$ behaves as

$e^{-2 \phi} \longrightarrow e^{-2 \phi}-\frac{N}{24} \int e^{2(\rho-\phi)} \int e^{-2(\rho-\phi)}\left(\frac{d z^{+}}{d y^{+}}\right)^{2}\left[\frac{d^{2}}{d z^{+2}} \log \frac{d y^{+}}{d z^{+}}-\frac{1}{2}\left(\frac{d}{d z^{+}} \log \frac{d y^{+}}{d z^{+}}\right)^{2}\right]$.

This anomalous transformation is just cancelled by the transformation law of the term $\frac{N}{12} M(\hat{\rho}-\hat{\phi})$ when $(\hat{\phi}, \hat{\rho})$ transform covariantly. This suggests the following generalization of the redefinitions (3.2), (3.3):

$$
\begin{gathered}
\rho-\phi=\hat{\rho}-\hat{\phi} \\
e^{-2 \phi}=e^{-2 \hat{\phi}}+\frac{N}{12} F(\hat{\phi})+\frac{N}{12} M(\hat{\rho}-\hat{\phi}),
\end{gathered}
$$

where $\mathrm{F}$ is an arbitrary function. It is clear that the new redefinitions still transform the non-covariant theory (3.1) into a covariant one. We must point out that the term $M(\hat{\rho}-\hat{\phi})$ is a purely gauge-dependent term since its argument is a conserved current. The equations of motion derived from the non-covariant action (3.1) are

$$
\begin{gathered}
\partial_{+} \partial_{-} e^{-2 \phi}+\lambda^{2} e^{2(\rho-\phi)}=0 \\
\partial_{+} \partial_{-}(\rho-\phi)=0, \\
\partial_{ \pm}^{2} e^{-2 \phi}-2 \partial_{ \pm}(\rho-\phi) \partial_{ \pm} e^{-2 \phi}+T_{ \pm \pm}^{f}+T_{ \pm \pm}^{W}=0 .
\end{gathered}
$$

The field redefinitions (3.6), (3.7) convert the above non-covariant equations into the covariant ones

$$
\begin{gathered}
\partial_{+} \partial_{-}\left(e^{-2 \hat{\phi}}+\frac{N}{12} F(\hat{\phi})\right)+\lambda^{2} e^{2(\hat{\rho}-\hat{\phi})}=0, \\
\partial_{+} \partial_{-}(\hat{\rho}-\hat{\phi})=0, \\
\partial_{ \pm}^{2}\left(e^{-2 \hat{\phi}}+\frac{N}{12} F(\hat{\phi})\right)-2 \partial_{ \pm}(\hat{\rho}-\hat{\phi}) \partial_{ \pm}\left(e^{-2 \hat{\phi}}+\frac{N}{12} F(\hat{\phi})\right) \\
-\frac{N}{12}\left[\left(\partial_{ \pm}(\hat{\rho}-\hat{\phi})\right)^{2}-\partial_{ \pm}^{2}(\hat{\rho}-\hat{\phi})\right]+T_{ \pm \pm}^{f}+T_{ \pm \pm}^{W}=0 .
\end{gathered}
$$


It is not difficult to check that this equations can be obtained from the following action

$$
S_{0}+S_{P}+\frac{N}{24 \pi} \int d^{2} x \sqrt{-g}\left[(\nabla \phi)^{2}-2 g^{\mu \nu} \nabla_{\mu} F(\phi) \nabla_{\nu} \phi+F(\phi) R-\phi R\right],
$$

which therefore defines a large family of 2D dilaton-gravity models parametrized by the arbitrary function F. Since the non-covariant theory is solvable the models (3.14) are automatically solvable. In the so-called "Kruskal" gauge $\hat{\rho}=\hat{\phi}$ the gauge dependent term $M(\hat{\rho}-\hat{\phi})$ vanishes and then the solutions to the semiclassical equations become

$$
e^{-2 \hat{\phi}}+\frac{N}{12} F(\hat{\phi})=e^{-2 \phi},
$$

where $\phi$ is the solution (3.4) of the non-covariant theory

$$
e^{-2 \phi}=e^{-2 \phi_{c l}}-\frac{N}{48} \log \left(-\lambda^{2} x^{+} x^{-}\right),
$$

$\phi_{c l}$ is the corresponding classical solution and $x^{ \pm}$are the coordinates defined by the gauge $\hat{\phi}=\hat{\rho}$. The models (1.7) can be recovered if one choose the function $\mathrm{F}$ as follows:

$$
F(\hat{\phi})=\frac{12}{N}\left(e^{-\frac{2}{n} \hat{\phi}}-e^{-2 \hat{\phi}}\right)+\frac{\hat{\phi}}{2 n} .
$$

Observe that the above choice modifies the classical action. Another natural choice for the function $\mathrm{F}$ is

$$
F(\hat{\phi})=a \hat{\phi}
$$

where $a$ is an arbitrary real parameter. In fact one can show that the above models are the only ones that can obtained from the procedure of Ref [5] by admitting a general form for the boundary conditions $T_{x^{ \pm} x^{ \pm}}^{W}$. The requirement of being able to rewrite the non-covariant semiclassical vacuum solutions in terms of the classical ones implies that $T_{x^{ \pm} x^{ \pm}}^{W}=-\frac{N a}{24} \frac{1}{\left(x^{ \pm}\right)^{2}}$. Hence one obtain the redefinitions $(3.6),(3.7)$ with $F(\hat{\phi})=a \hat{\phi}$. The semiclassical action (3.14) then becomes

$$
S_{0}+S_{P}+\frac{N}{24 \pi} \int d^{2} x \sqrt{-g}\left[(1-2 a)(\nabla \phi)^{2}+(a-1) \phi R\right] .
$$

This one-parameter class of models continuously interpolates between the RST model $\left(a=\frac{1}{2}\right)$ and the recently introduced model of Bose-Parker-Peleg [6] (BPP-model) $(a=0)$ which describes an evaporating black hole having a remnant as the semiclassical end-state geometry. The later model has recently emerged in the semiclassical limit of the non-perturbative approach of Ref. [11.

To finish this section let us briefly mention that the general semiclassical action (3.14) can also be transformed to the Liouville theory (1.6). The field redefinitions that reduce (3.14) to (1.6) in conformal gauge are

$$
\Omega=\sqrt{k} F(\phi)+\frac{e^{-2 \phi}}{\sqrt{k}},
$$




$$
\chi=\sqrt{k} \rho+\sqrt{k}(F(\phi)-\phi)+\frac{e^{-2 \phi}}{\sqrt{k}},
$$

\section{The RST-BPP models}

In this section we shall briefly consider the gravitational collapse described by the one-parameter class of models (3.19) thus interpolating the analysis of [2] and [6]. First we shall study the solutions with no radiation at infinity $<T_{ \pm \pm}^{f}\left(\sigma^{ \pm}\right)>=0$. Taking into account (3.15), (3.16) with $F(\phi)=a \phi$ we find that the asymptotically Minkowski vacuum solution, in Kruskal gauge, is

$$
e^{-2 \phi}+\frac{N a}{12} \phi=-\lambda^{2} x^{+} x^{-}-\frac{N}{48} \log \left(-\lambda^{2} x^{+} x^{-}\right)+C,
$$

where $\mathrm{C}$ is an integration constant. In the coordinates $\sigma^{ \pm}$, we have

$$
e^{-2 \phi}+\frac{N a}{12} \phi=e^{2 \lambda \sigma}-\frac{N}{48} 2 \lambda \sigma+C .
$$

For $\sigma \longrightarrow \infty$ the expression (4.2) and the analogue one for the metric

$$
e^{-2 \rho}+\frac{N a}{12} e^{-2 \lambda \sigma}(\rho-\lambda \sigma)=1-e^{-2 \lambda \sigma}\left(\frac{N}{48} 2 \lambda \sigma+C\right)
$$

approach to the linear dilaton vacuum. Let us now consider the solution describing a collapsing shell of matter $T_{++}^{f}=\frac{m}{\lambda x_{0}^{+}} \delta\left(x^{+}-x_{0}^{+}\right)$in Kruskal coordinates

$$
\begin{aligned}
e^{-2 \phi}+\frac{N a}{12} \phi= & -\lambda^{2} x^{+} x^{-}-\frac{N}{48} \log \left(-\lambda^{2} x^{+} x^{-}\right) \\
& -\frac{m}{\lambda x_{0}^{+}}\left(x^{+}-x_{0}^{+}\right) \theta\left(x^{+}-x_{0}^{+}\right)+C,
\end{aligned}
$$

where $\theta(x)$ is the step function. For $x^{+}>x_{0}^{+}$we have an evaporating black hole solution with a curvature singularity lying on the critical line $\left(\Omega^{\prime}=0\right)$

$$
\alpha=-\lambda^{2} x^{+}\left(x^{-}+\Delta\right)-\frac{N}{48} \log \left(-\lambda^{2} x^{+} x^{-}\right)+\frac{m}{\lambda}+C,
$$

where $\Delta=\frac{m}{\lambda^{3} x_{0}^{+}}$and $\alpha=\frac{N a}{24}\left(1-\log \frac{N a}{24}\right)$. The critical line initially lies behind an apparent horizon $\partial_{+} e^{-2 \phi}=0$, which is given by the curve

$$
-\lambda^{2} x^{+}\left(x^{-}+\Delta\right)=\frac{N}{48} .
$$

Due to Hawking radiation the apparent horizon recedes and meets the curvature singularity in a finite proper time. The intersecting point is located at

$$
\begin{gathered}
x_{i n t}^{-}=\frac{-\Delta}{1-\frac{N}{48} \exp \left[-\frac{48}{N}\left(\frac{m}{\lambda}+C-\alpha\right)+1\right]}, \\
x_{i n t}^{+}=\frac{1}{\lambda^{2} \Delta}\left\{\exp \left[\frac{48}{N}\left(\frac{m}{\lambda}+C-\alpha\right)+1\right]-\frac{N}{48}\right\},
\end{gathered}
$$


after which the singularity becomes naked. Following the reasoning of [2, 6] we shall try to construct a stable solution which continuously matches the evaporating black hole solution on the null curve $x^{-}=x_{i n t}^{-}$. A static solution in the asymptotically flat coordinates $\lambda \hat{\sigma}^{+}=\log \lambda x^{+}, \lambda \hat{\sigma}^{-}=-\log \left(-\lambda\left(x^{-}+\Delta\right)\right)$ is given by

$$
e^{-2 \rho}+\frac{N a}{12} \rho=-\lambda^{2} x^{+}\left(x^{-}+\Delta\right)-\frac{N}{48} \log \left(-\lambda^{2} x^{+}\left(x^{-}+\Delta\right)\right)+\hat{C} .
$$

The solution (4.4) can be matched to the solution (4.9) if the constant $\hat{C}$ is

$$
\hat{C}=-\frac{N}{48}\left(1-\log \frac{N}{48}\right)+\alpha .
$$

The constraint equations imply the existence of a shock wave originated at the end-point

$$
T_{--}^{f}\left(\hat{\sigma}^{-}\right)=\frac{N \lambda \Delta}{48 x_{\text {int }}^{-}} \delta\left(\hat{\sigma}^{-}-\hat{\sigma}_{\text {int }}^{-}\right)
$$

which account for the energy conservation. The energy radiated by the black hole is

$$
\begin{aligned}
& E_{\text {rad }}=\int_{-\infty}^{\hat{\sigma}_{\text {int }}^{-}}<T_{--}^{f}\left(\hat{\sigma}^{-}\right)>d \hat{\sigma}^{-}= \\
& m+\lambda C-\lambda \alpha-\frac{N \lambda}{48}\left(\log \frac{N}{48}-1\right)-\frac{N \lambda \Delta}{48 x_{i n t}^{-}}
\end{aligned}
$$

and the ADM mass of any static solution (4.9) can be obtained from the expression [12]

$$
M_{A D M}=\left.2 e^{2 \sigma}\left(\partial_{\sigma}+1\right)\left(\delta \phi-\delta \phi^{2}\right)\right|_{\sigma=\infty}
$$

One find that

$$
M_{A D M}=\lambda\left(C-C_{0}\right)
$$

where $C_{0}$ corresponds to an arbitrary reference solution. The unradiated mass before the end-point

$$
\frac{N \lambda}{48}\left(\log \frac{N}{48}-1\right)-\lambda C_{0}+\lambda \alpha+\frac{N \lambda \Delta}{48 x_{i n t}^{-}},
$$

coincides with the ADM mass of the remnant $\left(\lambda\left(\hat{C}-C_{0}\right)\right)$ plus the energy of the "thunderpop" 4.11). Like the BPP model the natural solution is $C_{0}=\hat{C}$, for which the remnant solution turns out to be the ground state. However, unlike the BPP model, the end-state geometry is not a semiinfinite throat. The left boundary of the end-state geometry is achieved at the critical points, for which $\Omega^{\prime}=0\left(\right.$ i.e,$\left.\sigma_{c r}=\frac{1}{2} \log \frac{N}{48}\right)$. In the proximities of the boundary $\sigma=\epsilon+\sigma_{c r}$ the metric behaves as

$$
d s^{2} \sim \frac{e^{2 \epsilon \frac{N}{48}}}{\frac{N a}{24}+\frac{N a}{12} \sqrt{\frac{a}{2}} \epsilon+\Theta\left(\epsilon^{2}\right)}\left(-d t^{2}+d \sigma^{2}\right),
$$

It is not difficult to show that the end-state geometry is still regular at the boundary although the space-time is no longer geodesically complete. 


\section{Towards a solvable model for a radiating $4 \mathrm{D}$ black hole}

The aim of this section is to extend our previous analysis of the CGHS model to a more general class of $2 \mathrm{D}$ dilaton gravity theories. In particular we want to generalize the field redefinitions (3.6), (3.7) to generate a semiclassical (solvable) model describing an evaporating spherically symmetric black hole. The clue for doing this is to realize that the field redefinitions (3.6), (3.7) of the CGHS model can be rewritten in the form

$$
\begin{gathered}
\rho-\phi=\hat{\rho}-\hat{\phi} \\
-\int^{y^{+}} e^{2(\rho-\phi)} \int e^{-2(\rho-\phi)} G_{++}-\int^{y^{-}} e^{2(\rho-\phi)} \int e^{-2(\rho-\phi)} G_{--}= \\
-\int^{y^{+}} e^{2(\hat{\rho}-\hat{\phi})} \int e^{-2(\hat{\rho}-\hat{\phi})} \hat{G}_{++}-\int^{y^{-}} e^{2(\hat{\rho}-\hat{\phi})} \int e^{-2(\hat{\rho}-\hat{\phi})} \hat{G}_{--} \\
+\frac{N}{12} F(\hat{\phi})+\frac{N}{12} M(\hat{\rho}-\hat{\phi}),
\end{gathered}
$$

where $G_{ \pm \pm}$are the null components of the Einstein tensor of the CGHS model

$$
G_{ \pm \pm}=e^{-2 \phi}\left(2 \partial_{ \pm}^{2} \phi-4 \partial_{ \pm} \rho \partial_{ \pm} \phi\right)
$$

and $\hat{G}_{ \pm \pm}=G_{ \pm \pm}(\hat{\rho}, \hat{\phi})$. Moreover the basic properties of the field redefinitions are maintained if we generalize the above expressions, for an arbitrary model, in the following way

$$
\begin{gathered}
j=\hat{j}, \\
-\int^{y^{+}} e^{2 j} \int e^{-2 j} G_{++}-\int^{y^{-}} e^{2 j} \int e^{-2 j} G_{--}= \\
-\int^{y^{+}} e^{2 \hat{j}} \int e^{-2 \hat{j}} \hat{G}_{++}-\int^{y^{-}} e^{2 \hat{j}} \int e^{-2 \hat{j}} \hat{G}_{--} \\
+\frac{N}{12} F(\hat{\phi})+\frac{N}{12} M(\hat{j}),
\end{gathered}
$$

where $j$ is a conserved current, $\partial_{+} \partial_{-} j=0$, which transforms as the conformal factor of the metric

$$
\begin{gathered}
j=\rho+\text { scalar terms } \\
M(\hat{j})=\hat{j}+\int^{y^{+}} e^{2 \hat{j}} \int e^{-2 \hat{j}}\left(\partial_{y^{+}} \hat{j}\right)^{2}+\int^{y^{-}} e^{2 \hat{j}} \int e^{-2 \hat{j}}\left(\partial_{y^{-}} \hat{j}\right)^{2},
\end{gathered}
$$

and

$$
G_{ \pm \pm}=T_{ \pm \pm}^{f}
$$

are the classical constrained equations of the model. 
For spherically symmetric gravity (1.8) the constrained equations with the Weyl-effective action are

$$
e^{-2 \phi}\left[2 \partial_{ \pm}^{2} \phi-4 \partial_{ \pm} \rho \partial_{ \pm} \phi-2\left(\partial_{ \pm} \phi\right)^{2}\right]=T_{ \pm \pm}^{f}+T_{ \pm \pm}^{W},
$$

and the remaining equations read as

$$
\begin{gathered}
\partial_{+} \phi \partial_{-} \phi-\partial_{+} \partial_{-} \phi+\partial_{+} \partial_{-} \rho=0, \\
8 \partial_{+} \phi \partial_{-} \phi-4 \partial_{+} \partial_{-} \phi+e^{2(\rho+\phi)}=0 .
\end{gathered}
$$

From the above equations it is easy to see that the analogue of the CGHS current $j=\rho-\phi$ is now

$$
j=\rho-\frac{1}{2} \phi-\frac{\lambda^{2}}{8} \int^{y^{+}} \int^{y^{-}} e^{2(\rho+\phi)} .
$$

Due to the non-local term this current should be finally defined by fixing its value on the Minkowski background $\left(d s^{2}=-d \sigma^{+} d \sigma^{-}, \frac{e^{-2 \phi}}{\lambda}=\frac{\sigma^{+}-\sigma^{-}}{2}\right)$. Following the analogy with the CGHS model the natural choice is then

$$
\left.j\right|_{\text {Minkowski }}=\frac{\lambda}{2}\left(\sigma^{+}-\sigma^{-}\right) \text {. }
$$

Let us now discuss the possible choices for the function F. The model which preserves the classical vacuum, i.e, the analogue of the RST model for spherically symmetric gravity, is given by the condition

$$
F(\phi)+\left.M(j)\right|_{\text {Minkowski }}=0 .
$$

A straightforward computation implies that

$$
F(\phi)=-\frac{1}{2} e^{-\phi}
$$

The simplest choice is however

$$
F(\phi)=0,
$$

which define the analogue of the BPP model for spherically symmetric gravity. Defining the "Kruskal" gauge $\left\{x^{ \pm}\right\}$by the condition $j=0$ we also have the relation $\lambda x^{ \pm}= \pm e^{ \pm \lambda \sigma^{ \pm}}$) In this gauge the equations of motion of the covariant model coincides with those of the non-covariant theory:

$$
\begin{aligned}
& \partial_{+} \hat{\phi} \partial_{-} \hat{\phi}-\partial_{+} \partial_{-} \hat{\phi}+\partial_{+} \partial_{-} \hat{\rho}=0, \\
& 8 \partial_{+} \hat{\phi} \partial_{-} \hat{\phi}-4 \partial_{+} \partial_{-} \hat{\phi}+\lambda^{2} e^{2(\hat{\rho}+\hat{\phi})}=0, \\
& e^{-2 \hat{\phi}}\left[2 \partial_{ \pm}^{2} \phi-4 \partial_{ \pm} \hat{\rho} \partial_{ \pm} \hat{\phi}-2\left(\partial_{ \pm} \hat{\phi}\right)^{2}\right]=T_{ \pm \pm}^{f}-\frac{N}{48} \frac{1}{\left(x^{ \pm}\right)^{2}}
\end{aligned}
$$

and therefore the study of the semiclassical equations can be reduced to that of the classical ones with an effective stress tensor of the form $T_{ \pm \pm}^{f}-\frac{N}{48} \frac{1}{\left(x^{ \pm}\right)^{2}}$. We have to remark that, in the absence of matter $T_{ \pm \pm}^{f}=0$, the above equations admit static solutions in the "Minkowskian" coordinates $\sigma^{ \pm}$. This consistence condition is a direct consequence of the choice (5.12) in the definition of the non-local conserved current (5.11). 


\section{Conclusions}

In this work we have elaborated a new scheme to generate solvable models of the CGHS theory in the semiclassical approximation. The solvability of the covariant one-loop corrected models (3.14) comes from the fact that all of them can be converted, via suitable field redefinitions, into a unique non-covariant one-loop theory (3.1) which inherits the solvability of the classical theory. The structure of the field redefinitions admit a natural and consistent generalization to more general dilaton-gravity models. We have considered the relevant case of spherically symmetric gravity and we have defined the analogue of the RST and $\mathrm{BPP}$ models. In the later case the semiclassical equations reduce, in a particular coordinate system, to the classical ones with an effective, $\rho$-independent stress tensor.

\section{Acknowledgements}

We would like to thank M. Navarro and C. F. Talavera for useful discussions.

\section{References}

[1] A. Bilal and C. Callan, Nucl. Phys. B 394 (1993) 73 ; S. de Alwis, Phys Lett. B 289 (1992) 278

[2] J. R. Russo, L. Susskind and L. Thorlacius, Phys. Rev D 46 (1993) 3444 ; D 47 (1993) 533

[3] C. G. Callan, S. B. Giddings, J. A. Harvey and A. Strominger, Phys. Rev. D 45 (1992) 1005

[4] A. Fabbri and J. G. Russo, " Solvable models in 2D dilaton gravity" CERN-TH / 95-267, hep-th / 9510109

[5] J. Navarro-Salas, M. Navarro and C. F. Talavera, Phys. Lett. B 356 (1995) 217

[6] S. Bose, L. Parker and Y. Peleg, Phys. Rev. D 52 (1995) 3512

[7] D. Karakhaniyan, R. Manvelyan and R. Mkrtchyan, Phys. Lett. B329 (1994) 185

[8] R. Jackiw, " Another view on massless matter-gravity fields in two dimensions ", hep-th / 9501016

[9] D. Amelino-Camelia, D. Back and D. Seminara, Phys. Lett. B 354 (1995) 213 ; D. Amelino-Camelia and A. Seminara, "Black hole radiation (with and) without Weyl anomaly", hep-th /9506128

[10] H. S. La, " Area-preserving Diffeomorphisms and 2-D Gravity " MIT-CTP2462, hep-th / 951014 
[11] A. Mikovic, "Unitary Theory of Evaporating 2D Black Holes", Imperial /TP/94-95/50 ;Phys. Lett. B 355 (1995) 85

[12] A. Bilal and I. Kogan, Phys. Rev. D 47 (1993) 5408 